\title{
AN APPLICATION OF THE CALCULUS OF VARIATIONS TO BOUNDARY VALUE PROBLEMS*
}

\author{
BX \\ A. O. HICKSON \\ INTRODUCTION
}

In a paper published in 1910 R. G. D. Richardson considered the Jacobi condition of the calculus of variations and, by means of well known theorems from integral equation theory and the calculus of variations, proved certain existence theorems and oscillation properties for the solutions of a restricted type of ordinary linear differential equations of the second order which contain a parameter. $\dagger$

Having set up the integral

$$
D(u)=\int_{0}^{1}\left[p(x) u^{\prime 2}-q(x) u^{2}\right] d x \quad\left(u^{\prime}=\frac{d u}{d x}\right),
$$

where $p$ and $q$ are analytic functions of $x$ in the interval $0 \leqq x \leqq 1, p$ being positive and $q$ nowhere positive within the interval, he considered the problem of finding in a class of arcs satisfying the conditions

$$
u(0)=0, \quad u(1)=0, \quad \int_{0}^{1} k(x) u^{2} d x=1,
$$

where $k$ is an analytic function of $x$ on the interval $0<x<1$, one for which $D(u)$ takes on a minimum value. The Euler-Lagrange differential equation of this isoperimetric problem was found to be

$$
L_{1}(u)=\frac{d}{d x}\left(p u^{\prime}\right)+q u+\lambda k u=0 .
$$

The function $k$ was first supposed to be not negative for $0 \leqq x \leqq 1$, and it then followed from the Hilbert theory of the integral equation corresponding to the boundary value problem defined by (2) and the first two equa-

* Presented to the Society, April 6, 1928; received by the editors November 5, 1928.

$\dagger$ †. G. D. Richardson, Das Jacobische Kriterium der Variationsrechnung und die Oszillationseigenschaften linearer Differentialgleichungen 2. Ordnung, Mathematische Annalen, vol. 68 (1910), p. 279.

$\ddagger$ The case where $k$ changes sign in the interval was also treated. 
tions of (1), that there exists a definitely increasing sequence of values $\lambda_{i}(i=1,2, \cdots)$ for each of which the equation $L_{1}(u)=0$ possesses a unique solution $U_{i}$ which satisfies the conditions imposed in the isoperimetric problem. Again making use of the theory of integral equations he was able to prove that in the class of functions $u(x)$ satisfying the conditions

$$
\begin{aligned}
& u(0)=0, \quad u(1)=0, \quad \int_{0}^{1} k(x) u^{2} d x=1, \\
& \int_{0}^{1} k U_{1} u d x=0, \quad \int_{0}^{1} k U_{2} u d x=0, \cdots, \quad \int_{0}^{1} k U_{n-1} u d x=0,
\end{aligned}
$$

the integral $D(u)$ has a minimum value and this value is the constant $\lambda_{n}$ described above. Furthermore the minimizing function giving $D(u)$ the value $\lambda_{n}$ is $u(x)= \pm U_{n}(x)$. By an application of some known results from the theory of differential equations and the Jacobi condition of the calculus of variations, it was then proved that $U_{i}$ vanishes $i-1$ times in the interval $0<x<1$.

Professor G. A. Bliss suggested the consideration of the above problem without recourse to integral equation theory. As a result of his suggestions a method was developed which made use of known theorems in the calculus of variations and which, in some respects, was readily applied to a more general case.

In this paper the boundary value problem is considered which arises from the problem of minimizing the second variation of the problem of the calculus of variations for a space of $n+1$ dimensions. This second variation is

where

$$
I_{2}(\eta)=\int_{x_{1}}^{x_{2}} 2 \omega_{0}\left(x, \eta, \eta^{\prime}\right) d x
$$

$$
2 \omega_{0}\left(x, \eta, \eta^{\prime}\right)=P_{i} \eta_{i} \eta_{j}+2 Q_{i} \eta_{i} \eta_{i}^{\prime}+R_{i} \eta_{i}^{\prime} \eta_{i}^{\prime} \quad(i, j=1, \cdots, n),
$$

and where the elements of $\eta_{i}$ form a set of so-called admissible variations. It is understood that in the formula for $2 \omega_{0}$ the summation convention of tensor analysis is used. A subscript $i$ or $j$ appearing twice in the same term indicates that the term is to be summed from 1 to $n$ with respect to that subscript. It is supposed that the quadratic form $R_{i j} \eta_{i}^{\prime} \eta_{i}^{\prime}$ is positive definite on the interval $x_{1} \leqq x \leqq x_{2}$ and that the coefficients of $2 \omega_{0}$ are continuous functions of $x$ with continuous second derivatives. In addition to the extension of Richardson's results to a space of $n+1$ dimensions, it will be noted that the integral $I_{2}(\eta)$ is of a less restricted form than the integral treated in his paper.

In $\S 1$ the matrix notation is introduced for the sake of brevity and 
simplicity of expression, and a few preliminary remarks are made. In $\$ 2$ we define the successive classes $H_{i}, H_{i}^{\prime}(i=1,2, \cdots)$ of $\operatorname{arcs} \eta(x)$ in which we are to consider the problem of minimizing $I_{2}(\eta)$. In $\S 3$ it is proved by methods of the calculus of variations that $I_{2}(\eta)$ has a greatest lower bound $\lambda_{0}$ in the class $H_{0}^{\prime}$, and that in the class $H_{0}$ there exists a set of $r_{0}$ linearly independent arcs for which

$$
I_{2}(\eta)-\lambda_{0} \int_{x_{1}}^{x_{2}} \eta \eta d x=0 .
$$

In $\S 4$ it is proved that $I_{2}(\eta)$ has a minimum value $\lambda_{i}$ in the general class $H_{i}^{\prime}$, and that in the class $H_{i}$ there exists a set of $r_{i}$ linearly independent arcs for which

$$
I_{2}(\eta)-\lambda_{i} \int_{x_{1}}^{x_{2}} \eta \eta d x=0 .
$$

The third and fourth sections thus carry through an induction which enables one to arrive at a set of existence theorems for the boundary value problem with a parameter. These results are a generalization of the existence theorem in Richardson's paper. One would not expect to find a direct generalization of his oscillation theorem, but it is very probable that there exist results for a system of ordinary linear differential equations of the second order with a parameter, which would be somewhat analogous to his oscillation theorem.

The theorems from the calculus of variations to which one will refer in $\$ 4$ are contained in most convenient form in a set of lectures* on the Lagrange problem given by Professor Bliss at Chicago. Analogous theorems for the problem without adjoined differential equations will be used to obtain the results of $\S 3$.

In preparing this dissertation I have had the privilege of working under Professor Bliss and I wish to acknowledge his never failing advice and direction.

\section{NOTATIONS AND PRELIMINARY REMARKS}

The problem of the calculus of variations is that of finding among the $\operatorname{arcs}$

$$
y_{i}=y_{i}(x) \quad\left(i=1, \cdots, n ; x_{1} \leqq x \leqq x_{2}\right)
$$

which join two given points 1 and 2 in the $\left(x, y_{1}, \cdots, y_{n}\right)$-space, one which minimizes an integral of the form

* G. A. Bliss, The Problem of Lagrange in the Calculus of Variations, a mimeographed record of a set of lectures given at the University of Chicago in the summer of 1925. (Reference A.) See also Bolza, Vorlesungen über Variationsrechnung, pp. $542 \mathrm{ff}$. 


$$
I_{0}=\int_{x_{1}}^{x_{2}} f\left(x, y, y^{\prime}\right) d x,
$$

where $y$ and $y^{\prime}$ are symbols for the vectors $\left(y_{1}, \cdots, y_{n}\right)$ and $\left(y_{1}^{\prime}, \cdots, y_{n}^{\prime}\right)$ respectively.

The matrix and vector notation will be used. The symbols

$$
y, z, u, v, J, \xi, \eta, \zeta, \mu, \phi
$$

will always represent vectors. Italic capitals, unless otherwise stated, will symbolize matrices. Any small symbol with a bracketed or double superscript will always represent a vector and any symbol with a subscript will stand for a single element. The meaning of other symbols which may occur will be clear from the context. If, for example, $Q$ is a matrix and $\eta$ a vector in this simplified notation, then $Q \eta$ represents a vector $\left(Q_{i 1} \eta_{1}+\cdots+Q_{i n} \eta_{n}\right.$; $i=1, \cdots, n)$ and $\eta Q$ a vector $\left(\eta_{1} Q_{1 i}+\cdots+\eta_{n} Q_{n i} ; i=1, \cdots, n\right)$, which shows that $Q \eta=\eta \bar{Q}$ where $\bar{Q}$ denotes the transpose of $Q$. We have, in fact, the relations

$$
Q \eta \eta^{\prime}=(Q \eta) \eta^{\prime}=\eta^{\prime}(Q \eta)=\left(\eta^{\prime} Q\right) \eta=\bar{Q} \eta^{\prime} \eta=(\eta \bar{Q}) \eta^{\prime}=\eta\left(\bar{Q} \eta^{\prime}\right)
$$

The expression $\eta \xi$ will often be used for $I \eta \xi$ where $I$ is the identity matrix.*

The assumption will be made as usual that in a neighborhood $N$ of the values $\left(x, y, y^{\prime}\right)$ on the minimizing arc the function $f$ has continuous derivatives up to and including those of the fourth order. An admissible arc is defined to be an arc of the form (3) which is continuous and consists of a finite number of arcs each of which possesses a continuous derivative. A variation arc $\eta(x)$ must be admissible.

The second variation $\dagger$ of the integral $I_{0}$ is

where

$$
I_{2}(\eta)=\int_{x_{1}}^{x_{2}} 2 \omega_{0}\left(x, \eta, \eta^{\prime}\right) d x
$$

$$
2 \omega_{0}\left(x, \eta, \eta^{\prime}\right)=f_{y y} \eta \eta+2 f_{y^{\prime} y} \eta \eta^{\prime}+f_{y^{\prime} y^{\prime}} \eta^{\prime} \eta^{\prime} \text {. }
$$

Writing $P=f_{y y}, Q=f_{y^{\prime} y}$ and $R=f_{y^{\prime} y^{\prime}}$, one has

$$
I_{2}(\eta)=\int_{x_{1}}^{x_{2}}\left(P \eta \eta+2 Q \eta \eta^{\prime}+R \eta^{\prime} \eta^{\prime}\right) d x
$$

* For a more detailed explanation of the matrix notation see G. A. Bliss, Annals of Mathematics, (2), vol. 6 (1905), p. 58.

† J. Hadamard, Leçons sü le Calcul des Variations, vol. 1 p. 317. 
On account of the assumptions made concerning the function $f$, the matrices $P, Q$ and $R$ are continuous and have continuous derivatives of the first two orders. It will be also assumed that the quadratic form $R \eta \eta$ is positive definite on $x_{1} \leqq x \leqq x_{2}$. Then the determinant of the matrix $R$ will be different from zero on that interval.

If $E_{12}$ is a minimizing arc, then $I_{2}(\eta)$ must be greater than or equal to zero for every set of admissible variations satisfying the end conditions $\eta\left(x_{1}\right)$ $=\eta\left(x_{2}\right)=0$. This result suggests a new minimum problem in the $x \eta$-space with the integral $I_{2}$ in place of $I_{0}$ and the end points $(x, \eta)=\left(x_{1}, 0\right),(x, \eta)$ $=\left(x_{2}, 0\right)$ in place of the end points of $E_{12}$. By a solution of a system of ordinary linear homogeneous differential equations of the second order will be meant an arc, having continuous first and second derivatives, defined by a vector $\eta(x)$ which satisfies these equations. The class $H_{0}$ is defined to be all admissible $\operatorname{arcs} \eta(x)$ for which $\eta\left(x_{1}\right)=\eta\left(x_{2}\right)=0$, and the problem in $x \eta$-space may be stated as that of minimizing $I_{2}(\eta)$ in the class of arcs $H_{0}$. Since $|R| \neq 0$ on $x_{1} \leqq x \leqq x_{2}$ and $\left|\omega_{0 \eta^{\prime} \eta^{\prime}}\right|=|R|$, it follows* that an arc which minimizes $I_{2}(\eta)$ in the class $H_{0}$ must be a solution of the system of equations

$$
J(\eta)=0, \quad \eta\left(x_{1}\right)=\eta\left(x_{2}\right)=0,
$$

where

$$
J(\eta)=\frac{d}{d x} \omega_{0 \eta^{\prime}}-\omega_{0 \eta}=\frac{d}{d x}\left(Q \eta+R \eta^{\prime}\right)-\left(P \eta+\bar{Q}_{\eta^{\prime}}\right) .
$$

The system of equations (4) is linear and homogeneous in the variables $\eta, \eta^{\prime}, \eta^{\prime \prime}$ and the determinant of the variables $\eta^{\prime \prime}$ is the determinant of the matrix $\omega_{0 \eta^{\prime} \eta^{\prime}}$, which is equal to the determinant of $R$. The determinant $|R|$ is different from zero along $E_{12}$ and hence from the theory of differential equations it follows that the system (4) has one and but one solution $\eta$ taking prescribed values of $\eta, \omega_{0 \eta^{\prime}}$ at a given value of $x$. A matrix of $n$ solutions $u^{(k)}(k=1, \cdots, n)$ exists therefore for which the matrix $U=\left\|u^{(k)}\right\|$ is the identity matrix and the corresponding matrix of the functions $\omega_{0 \eta^{\prime}}$ has all its elements zero at the point 1 . Also there exists a matrix of $n$ solutions $v^{(k)}(k=1, \cdots, n)$ for which the matrix $V=\left\|v^{(k)}\right\|$ has all its elements zero at the point 1 and the corresponding matrix of the functions $\omega_{0 \eta^{\prime}}$ is the identity matrix at that point. Furthermore the columns of $U$ and $V$ form $2 n$ linearly independent solutions of the system (4). If $u$ and $v$ are two solutions of (4) for which the constant expression

$$
u \omega_{0 v}-v \omega_{0 u}
$$

* Hadamard, loc. cit., p. 67, p. 73. 
is zero, they are said to be conjugate solutions. ${ }^{*}$ The solutions $u^{(k)}(k=1$, $\cdots, n)$ are conjugate pairs, since their functions $\omega_{0 \eta^{\prime}}$ all vanish for $x=x_{1}$.

\section{The Classes of ARCS $H_{i}$ AND $H_{i}^{\prime}$}

We will now define the classes of arcs for the various minimum problems to be studied and will sketch, as far as is necessary for that purpose, the methods of the next two sections. The class of $\operatorname{arcs} H_{0}$ is by definition made up of all arcs $\eta(x)$ which satisfy the conditions $\eta\left(x_{1}\right)=0, \eta\left(x_{2}\right)=0$ and which possess continuous derivatives on the interval $x_{1} \leqq x \leqq x_{2}$ except possibly at a finite number of corners, and the class $H_{0}^{\prime}$ is the totality of arcs of the class $H_{0}$ which satisfy the further condition

$$
\int_{x_{1}}^{x_{2}} \eta \eta d x=1
$$

Arcs which satisfy this condition will be called normed. The problem of minimizing $I_{2}(\eta)$ in the class $H_{0}^{\prime}$ will be considered in the next section. It will be proved that $I_{2}(\eta)$ has a minimum value $\lambda_{0}$ in the class $H_{0}^{\prime}$, and that there exists a set of linearly independent $\operatorname{arcs} \eta^{0 k}\left(k=1, \cdots, r_{0}\right)$ in the class $H_{0}$ such that

$$
I_{2}(\eta)-\lambda_{0} \int_{x_{1}}^{x_{2}} \eta \eta d x=0 .
$$

The functions $\eta^{0 k}\left(k=1, \cdots, r_{0}\right)$ are called the characteristic functions for the class $H_{0}$. The class $H_{1}$ is now defined to be all those arcs of the class $H_{0}$ for which the conditions

$$
\int_{x_{1}}^{x_{2}} \eta^{0 k} \eta d x=0 \quad\left(k=1, \cdots, r_{0}\right)
$$

are satisfied. The class $H_{1}^{\prime}$ is then taken to be all normed arcs of the class $H_{1}$. By methods developed in $\S 3$ it is possible to prove directly that there exists a set of linearly independent characteristic functions $\eta^{1 k}\left(k=1, \cdots, r_{1}\right)$ in the class $H_{1}$ for which

$$
I_{2}(\eta)-\lambda_{1} \int_{x_{1}}^{x_{2}} \eta \eta d x=0
$$

where $\lambda_{1}$ is the greatest lower bound of the values of $I_{2}(\eta)$ in the class $H_{1}^{\prime}$. The class $H_{2}$ is then defined to be all arcs of $H_{1}$ for which

$$
\int_{x_{1}}^{x_{2}} \eta^{1 k} \eta d x=0 \quad\left(k=1, \cdots, r_{1}\right),
$$

and the class $H_{2}^{\prime}$ consists of all normed arcs of the class $H_{2}$.

\footnotetext{
* Bliss, loc. cit. A, p. 73; von Escherich, Wiener Berichte, vol. 107, p. 1244.
} 
Supposing the existence of a set of characteristic functions in the class $\boldsymbol{H}_{2}$, one defines the classes $H_{3}$ and $\boldsymbol{H}_{3}^{\prime}$ in a manner similar to the definitions of the classes $H_{2}$ and $H_{2}^{\prime}$. One proceeds in this manner and defines the general classes $H_{i}$ and $H_{i}^{\prime}$, the class $H_{i}$ being all those arcs in the class $H_{i-1}$ for which

$$
\int_{x_{1}}^{x_{2}} \eta^{i-1, k} \eta d x=0 \quad\left(k=1, \cdots, r_{i-1}\right)
$$

where, if $\lambda_{i-1}$ is the minimum value of $I_{2}(\eta)$ in the class $H_{i-1}^{\prime}$, the $\eta^{i-1, k}$ are the $r_{i-1}$ linearly independent characteristic functions in the class $H_{i-1}$ for which

$$
I_{2}(\eta)-\lambda_{i-1} \int_{x_{1}}^{x_{2}} \eta \eta d x=0 .
$$

The class $H_{i}^{\prime}$ consists of all normed arcs of the class $H_{i}$. That is, the class $H_{i}^{\prime}$ is made up of all arcs $\eta$ which have continuous derivatives except at a finite number of points on the interval $x_{1} \leqq x \leqq x_{2}$ and which satisfy the conditions

$$
\begin{gathered}
\eta\left(x_{1}\right)=\eta\left(x_{2}\right)=0, \\
\int_{x_{1}}^{x_{2}} \eta \eta d x=1, \\
\int_{x_{1}}^{x_{2}} \eta^{0 k} \eta d x=0\left(k=1, \cdots, r_{0}\right), \cdots, \int_{x_{1}}^{x_{2}} \eta^{i-1, k} \eta d x=0\left(k=1, \cdots, r_{i-1}\right) .
\end{gathered}
$$

3. The Minimum of the SECOND variation in the Class $\boldsymbol{H}_{0}^{\prime}$

The problem of minimizing $I_{2}(\eta)$ in the class $H_{0}^{\prime}$ is that of finding among all admissible $\operatorname{arcs} \eta$ satisfying the conditions

$$
\int_{x_{1}}^{x_{2}} \eta \eta d x=1, \quad \eta\left(x_{1}\right)=\eta\left(x_{2}\right)=0
$$

one for which the integral $I_{2}(\eta)$ takes a minimum value. If one introduces the new variable

$$
\zeta_{0}=\int_{x_{1}}^{x_{2}} \eta \eta d x
$$

the problem becomes that of finding among the $\operatorname{arcs} \eta(x), \zeta_{0}(x)$ joining the two points $\left(x, \eta, \zeta_{0}\right)=\left(x_{1}, 0,0\right)$ and $\left(x, \eta, \zeta_{0}\right)=\left(x_{2}, 0,1\right)$ and satisfying the equation

$$
\phi_{0} \equiv \zeta_{0}^{\prime}-\eta \eta=0
$$

one which minimizes the integral

$$
I_{2}(\eta)=\int_{x_{1}}^{x_{2}} 2 \omega_{0}\left(x, \eta, \eta^{\prime}\right) d x
$$


That is, the isoperimetric problem has been replaced by an equivalent Lagrange problem of the calculus of variations. Having set up the form

$$
\Omega\left(x, \eta, \eta^{\prime}\right)=2 \omega_{0}+\lambda\left(\zeta_{0}^{\prime}-\eta \eta\right)
$$

one notes that $\left|\Omega_{\eta^{\prime} \eta^{\prime}}\right|$ is different from zero on $x_{1} \leqq x \leqq x_{2}$. The first necessary condition* of the Lagrange problem gives then the following result:

TheORem 1. An arc $\eta(x)$ which minimizes $I_{2}(\eta)$ in the class $H_{0}^{\prime}$ must be a solution of the boundary value problem

$$
J(\eta)+\lambda \eta=0, \quad \eta\left(x_{1}\right)=\eta\left(x_{2}\right)=0 .
$$

It is further possible to prove

THEOREM 2. The values of the integral $I_{2}(\eta)$ in the class $H_{0}^{\prime}$ have a greatest lower bound $\lambda_{0}$.

To prove this a new vector $\zeta$ is introduced by means of the $n$ equations

$$
\zeta=\omega_{0 \eta^{\prime}}=Q \eta+R \eta^{\prime} .
$$

Since the determinant of $R$ is different from zero on $x_{1} \leqq x \leqq x_{2}$, these equations may be solved for the elements of the vector $\eta^{\prime}$ in the form

$$
\eta^{\prime}=R^{-1} \zeta-R^{-1} Q \eta=\zeta R^{-1}-\eta \bar{Q} R^{-1} .
$$

One substitutes this expression for $\eta^{\prime}$ in the integral

$$
I_{2}(\eta)-\lambda \int_{x_{1}}^{x_{2}} \eta \eta d x=\int_{x_{1}}^{x_{2}}\left[(P-\lambda I) \eta \eta+2 Q \eta \eta^{\prime}+R \eta^{\prime} \eta^{\prime}\right] d x
$$

and finds that the integral takes the form

where $A=P-\bar{Q} R^{-1} Q$.

$$
\int_{x_{1}}^{x_{2}}\left[(A-\lambda I) \eta \eta+R^{-1} \zeta \zeta\right] d x,
$$

The matrix of the quadratic form $(A-\lambda I) \eta \eta$ is

$$
A-\lambda I=\left\|\begin{array}{llll}
a_{11}-\lambda & a_{12} & \cdots & a_{1 n} \\
a_{21} & a_{22}-\lambda & \cdots & a_{2 n} \\
\cdot & \cdot & \cdots & \cdot \\
a_{n 1} & \cdot & \cdots & a_{n n}-\lambda
\end{array}\right\|
$$

where the $a_{i j}$ are independent of $\lambda$. The chain of principal minors from $a_{11}-\lambda$ to the determinant of the matrix $A-\lambda I$ have respectively $-\lambda, \lambda^{2}, \ldots$, $(-1)^{n} \lambda^{n}$ as the dominant terms in $\lambda$. Certainly for $\lambda$ sufficiently large and

* Bliss, loc. cit. A, p. 10. 
negative these determinants will all be positive.* That is, for a sufficiently large negative value of $\lambda$ the form $(A-\lambda I) \eta \eta$ will be positive definite for all $x$ on $x_{1} \leqq x \leqq x_{2}$. Also under the transformation $u=R^{-1} \zeta \zeta$ the form $R u u$ becomes $R^{-1} \zeta \zeta$. Hence the assumption that $R u u$ is positive definite implies the same property for $R^{-1} \zeta \zeta$. There exists therefore a smallest value $\lambda_{0}$ of $\lambda$ such that

$$
I_{2}(\eta)-\lambda_{0} \int_{x_{1}}^{x_{2}} \eta \eta d x \geqq 0
$$

for every $\eta$ in the class $H_{0}$. Hence the theorem.

The following lemma is of importance in the proof of later theorems.

LEMMA 1. Along every admissible arc $\eta$ which is a solution of the boundary value problem

$$
J(\eta)+\lambda \eta=0, \quad \eta\left(x_{1}\right)=\eta\left(x_{2}\right)=0
$$

the integral

has the value zero.

$$
I_{2}(\eta)-\lambda \int_{x_{1}}^{x_{2}} \eta \eta d x
$$

For the proof one makes use of a well known property of quadratic forms to write the integral $I_{2}(\eta)$ as

$$
I_{2}(\eta)=\int_{x_{1}}^{x_{2}} 2 \omega_{0} d x=\int_{x_{1}}^{x_{2}}\left(\eta \omega_{0 \eta}+\eta^{\prime} \omega_{0 \eta^{\prime}}\right) d x .
$$

On integrating the second term of the right member by parts, this becomes

$$
I_{2}(\eta)=\left.\eta \omega_{0 \eta^{\prime}}\right|_{x_{1}} ^{x_{2}}+\int_{x_{1}}^{x_{2}} \eta\left(\omega_{0 \eta}-\frac{d}{d x} \omega_{0 \eta^{\prime}}\right) d x .
$$

If $\eta$ is a solution of the boundary value problem (5), one notes immediately that

$$
I_{2}(\eta)=\lambda \int_{x_{1}}^{x_{2}} \eta \eta d x
$$

COROLlARY. Along every normed admissible arc which is a solution of the boundary value problem (5) the integral $I_{2}(\eta)$ has the value $\lambda$.

\footnotetext{
* It is of interest to note that for a sufficiently large and positive $\lambda$ the principal minor determinants of the chain will alternate in sign. If $R \eta \eta$ is supposed negative definite on $x_{1} \leqq x \leqq x_{2}$, then the values of $I_{2}(\eta)$ have a least upper bound in the class $H_{0}^{\prime}$.
} 
The problem of minimizing the integral

$$
I_{2}(\eta)-\lambda_{0} \int_{x_{1}}^{x_{2}} \eta \eta d x
$$

in the class $H_{0}$ will now be considered. The arc $\eta=0$ is a minimizing arc. For if there existed an arc $u$ in $H_{0}$ for which the integral (6) were less than zero, then the vector

$$
\eta=u\left[\int_{x_{1}}^{x_{2}} u u d x\right]^{-1 / 2}
$$

would be in the class $H_{0}^{\prime}$ and would give $I_{2}(\eta)$ a value less than $\lambda_{0}$ which is a contradiction.

The second variation of (6) is

$$
\int_{x_{1}}^{x_{2}}\left(\Omega_{0 \eta \eta} \xi \xi+2 \Omega_{0 \eta^{\prime} \eta} \xi \xi^{\prime}+\Omega_{0 \eta^{\prime} \eta^{\prime}} \xi^{\prime} \xi^{\prime}\right) d x
$$

where

$$
2 \Omega_{0}\left(x, \eta, \eta^{\prime}\right)=\left(P-\lambda_{0} I\right) \eta \eta+2 Q \eta \eta^{\prime}+R \eta^{\prime} \eta^{\prime}
$$

and $\xi$ is a set of admissible variations. The Euler system and the Jacobi* system of $n$ differential equations for the problem of minimizing the integral (6) are therefore identical; namely

$$
J(\eta)+\lambda \eta=0
$$

for $\lambda=\lambda_{0}$.

THEOREM 3. There exists a set of $r_{0}$ linearly independent arcs $\eta^{0 k}(x)(k=1$, ..., $r_{0}$ ) in the class $H_{0}$ such that

$$
I_{2}\left(\eta^{0 k}\right)-\lambda_{0} \int_{x_{1}}^{x_{2}} \eta^{0 k} \eta^{0 k} d x=0 \quad\left(k=1, \cdots, r_{0}\right) .
$$

For the proof one considers the problem of minimizing the integral (6) in the class $H_{0}$. As noted above, the Euler and the Jacobi differential equations for this problem are identical. In $\$ 1$ the solutions of such a system were considered and a matrix of solutions $V$ was uniquely determined whose elements all vanish at $x=x_{1}$ and such that the matrix of the corresponding functions $\omega_{0 \eta^{\prime}}$ was the identity matrix $I$. Also if $\lambda$ is allowed to vary the solutions of the system (8) are continuous functions of $\lambda$. $\dagger$ The determinant

* Bliss, loc. cit. A, pp. 57-63. The minimum problem discussed above has no adjoined differential equations, and theorems, corresponding to those of the Lagrange problem, hold for this more special case.

† J. Horn, Gewöhnliche Differentialgleichungen beliebiger Ordnung, p. 293. 
of the matrix $V$ is a Mayer* determinant $\Delta\left(x, x_{1}, \lambda_{6}\right)$ for our minimizing problem, since its columns are $n$ linearly independent solutions of the Jacobi differential equations and its elements vanish at $x=x_{1}$. The vanishing of $\Delta\left(x, x_{1}, \lambda_{0}\right)$ for a value of $x$ between $x_{1}$ and $x_{2}$ implies that there exists an admissible arc such that the second variation (7) is less than zero.* Since (7) is the same as the integral (6), this result contradicts the previous statement that $\lambda_{0}$ is the minimum value of $I_{2}(\eta)$ in the class $H_{0}^{\prime}$. Hence $\Delta\left(x, x_{1}, \lambda_{0}\right)$ cannot vanish for a value $x$ between $x_{1}$ and $x_{2}$.

In order to prove that $\Delta\left(x, x_{1}, \lambda_{0}\right)$ must vanish at $x=x_{2}$, it will now be assumed that $\Delta\left(x, x_{1}, \lambda_{0}\right)$ is different from zero on $x_{1}<x \leqq x_{2}$ and it will then be shown that this leads to a contradiction. The determinant of the matrix $U$ with initial value $I$ at $x=x_{1}$, as set up in $\$ 1$, is a continuous function of $\lambda$ which is different from zero at $x=x_{1}$. Also the columns of $U$ form a conjugate system of solutions of the Jacobi differential equations. If $\lambda$ is allowed to vary, the matrix $U$ with the same initial conditions as before will still have for columns a conjugate system of solutions of the corresponding Jacobi differential equations, and for $h$ sufficiently small and positive there will be an interval $x_{1}-h \leqq x \leqq x_{1}+h$ on which $U\left(x, \lambda_{0}+\epsilon\right)$ will be different from zero. It is known $\dagger$ that $\Delta\left(x, x_{1}, \lambda_{0}+\epsilon\right)$ cannot vanish for a pair of points $x^{\prime}, x^{\prime \prime}$ on an interval $x_{1}-h \leqq x \leqq x_{1}+h$ for which there is a conjugate system with determinant $\left|U\left(x, \lambda_{0}+\epsilon\right)\right| \neq 0$. Thus $\Delta\left(x, x_{1}, \lambda_{0}+\epsilon\right)$ is different from zero on $x_{1}<x \leqq x_{1}+h$. From continuity considerations $\Delta\left(x, x_{1}, \lambda_{0}+\epsilon\right)$ is different from zero on $x_{1}+h \leqq x \leqq x_{2}$ if also $\Delta\left(x, x_{1}, \lambda_{0}\right)$ is different from zero on that interval, $\epsilon$ being positive and sufficiently small. That is, $\Delta\left(x, x_{1}, \lambda_{0}\right) \neq 0$ on $x_{1}<x \leqq x_{2}$ implies $\Delta\left(x, x_{1}, \lambda_{0}+\epsilon\right) \neq 0$ on $x_{1}<x \leqq x_{2}$ for $\epsilon$ positive and sufficiently small.

Consider now the problem of minimizing

$$
I_{2}(\eta)-\left(\lambda_{0}+\epsilon\right) \int_{x_{1}}^{x_{2}} \eta \eta d x
$$

in the class $H_{0}$. If $\Delta\left(x, x_{1}, \lambda_{0}+\epsilon\right)$ is different from zero on the interval $x_{1}<x \leqq$ $x_{2}$, there exists no conjugate point $\ddagger$ on this interval for the extremal $\operatorname{arc} \eta=0$. The Clebsch§ quadratic form is $R \pi \pi$ which is greater than zero for every set $\pi=\left(\pi_{1}, \cdots, \pi_{n}\right) \neq(0, \cdots, 0)$. The Weierstrass§ function $E\left(x, \eta, \eta^{\prime}\right.$, $\left.\zeta^{\prime}\right)$ is found to be

$$
R\left(\zeta^{\prime} \zeta^{\prime}-2 \eta^{\prime} \zeta^{\prime}+\eta^{\prime} \eta^{\prime}\right)=R\left(\zeta^{\prime}-\eta^{\prime}\right)\left(\zeta^{\prime}-\eta^{\prime}\right)
$$

* See first footnote on page 572.

$\dagger$ Bliss, loc. cit. A, p. 75; Bolza, loc. cit., p. 632.

$\ddagger$ Bliss, loc. cit. A, p. 52; Bolza, loc. cit., p. 610 .

$\S$ Bliss, loc. cit. A, pp. 49-51. 
which is greater than zero for every $\zeta^{\prime} \neq \eta^{\prime}$. From the sufficiency theorem* of the calculus of variations it therefore follows that the extremal arc $\eta=0$, containing no conjugate point on $x_{1}<x \leqq x_{2}$, gives (9) a smaller value than any other arc $\xi$ of the class $H_{0}$ which is not identically zero. If then $\Delta(x$, $\left.x_{1}, \lambda_{0}\right)$ is different from zero on $x_{1}<x \leqq x_{2}$, one finds that $\Delta\left(x, x_{1}, \lambda_{0}+\epsilon\right)$ has the same property and hence $I_{2}(\eta)>\lambda_{0}+\epsilon$ for every arc of the class $B_{0}^{\prime}$. This contradicts the hypothesis that $\lambda_{0}$ is the greatest lower bound of $I_{2}(\eta)$ in the class $H_{0}^{\prime}$. Therefore $\Delta\left(x_{2}, x_{1}, \lambda_{0}\right)=0$. That is, the columns of the matrix $V$ are linearly dependent at $x=x_{2}$.

If the rank of the matrix $V$ is $n-r_{0}$ at $x=x_{2}$, the equations

$$
V\left(x_{2}\right) c=0 \text {, }
$$

where $c=\left(c_{1}, \cdots, c_{n}\right)$ is independent of $x$, have $r_{0}$ linearly independent solutions $c \neq 0$ and these define a fundamental system of solutions of these linear equations in terms of which all other solutions are linearly expressible. Moreover for each such $c$ the vector $\eta=V c$ is a solution of the boundary value problem (5) corresponding to the value $\lambda=\lambda_{0}$. Hence by Lemma 1 there exist $r_{0}$ linearly independent $\operatorname{arcs} \eta^{0 k}\left(k=1, \cdots, r_{0}\right)$ of the class $B_{0}$ such that

$$
I_{2}(\eta)-\lambda_{0} \int_{x_{1}}^{x_{2}} \eta \eta d x=0 .
$$

CoRollary. There exists a set of $r_{0}$ linearly independent arcs in the class $H_{0}^{\prime}$ for which the integral $I_{2}(\eta)$ takes the value $\lambda_{0}$.

4. The minimum of the SEcond variation In the class $H_{i} ;$

In the previous section it has been proved that $I_{2}(\eta)$ has a minimum value $\lambda_{0}$ in the class $H_{0}^{\prime}$, and that there exists a set of $r_{0}$ linearly independent arcs in the class $H_{0}$ for which the relation

$$
I_{2}(\eta)-\lambda_{0} \int_{x_{1}}^{x_{2}} \eta \eta d x=0
$$

holds. The classes $H_{1}$ and $H_{1}^{\prime}$ will then be defined as in $\S 2$. Since $H_{1}^{\prime}$ is a sub-class of $H_{0}^{\prime}$ it follows that $I_{2}(\eta)$ has a greatest lower bound $\lambda_{1}$ in the class $H_{1}^{\prime}$ and that $\lambda_{1}$ cannot be less than $\lambda_{0}$. In order to prove by induction that there exists in the general class $H_{i}$ a set of $r_{i}$ linearly independent arcs for which the integral

$$
I_{2}(\eta)-\lambda_{i} \int_{x_{1}}^{x_{2}} \eta \eta d x
$$

* Bliss, loc. cit. A, p. 70; Bolza, loc. cit., p. 638. 
is zero, we will assume the existence of such a set for each of the classes $H_{1}$, $H_{2}, \cdots, B_{i-1}$ with $\lambda_{i}$ replaced by $\lambda_{1}, \lambda_{2}, \cdots, \lambda_{i-1}$, respectively, in the integral (11). We will denote these sets in the classes $H_{1}, \cdots, B_{i-1}$ by $\eta^{1 k}, \cdots, \eta^{i-1, k}$ where the range of $k$ will be determined by the first superscript. For example, in the symbol $\eta^{j k}, k$ has the range $1, \cdots, r_{j}$. The classes $H_{i}$ and $H_{i}$ will then be defined as in $\$ 2$. Since $H_{i}^{\prime}$ is a sub-class of $H_{i-1}^{\prime}$ it follows that $I_{2}(\eta)$ has a greatest lower bound $\lambda_{i}$ in the class $H_{i}^{\prime}$ and that $\lambda_{i}$ cannot be less than $\lambda_{i-1}$.

The problem of minimizing the integral (11) in the class $B_{i}$ will now be considered. If we introduce the $r_{0}+r_{1}+\cdots+r_{i-1}$ new variables

$$
\zeta_{0 k}=\int_{x_{1}}^{x} \eta^{0 k} \eta d x, \quad \zeta_{1 k}=\int_{x_{1}}^{x} \eta^{1 k} \eta d x, \cdots, \zeta_{i-1, k}=\int_{x_{1}}^{x} \eta^{i-1, k} \eta d x,
$$

set $\rho=r_{0}+r_{1}+\cdots+r_{i-1}$, write $\eta^{k}$ and $\zeta_{k}(k=1,2, \cdots, \rho)$ as new notations for the sets $\eta^{0 k}, \eta^{1 k}, \cdots, \eta^{i-1, k}$ and $\zeta_{0 k}, \zeta_{1 k}, \cdots, \zeta_{i-1, k}$, respectively, and let $z$ represent the vector whose elements are $\eta$ and the $\zeta_{k}$, the problem becomes that of finding among all the admissible $\operatorname{arcs} z(x)$ joining the two points $(x, z)=\left(x_{1}, 0\right)$ and $(x, z)=\left(x_{2}, 0\right)$, and satisfying the equations

$$
\phi_{k} \equiv \zeta_{k}^{\prime}-\eta^{k} \eta=0,
$$

one which gives the integral (11) a minimum value. The problem is therefore a Lagrange problem with $\rho$ adjoined differential equations. The $\operatorname{arc} z=0$ is a minimizing arc. For, if there existed a vector $u$ in the class $B_{i}$ for which the integral (11) were less than zero, then the arc

$$
\eta=u\left(\int_{x_{1}}^{x_{2}} u u d x\right)^{-1 / 2}
$$

would be in the class $H_{i}^{\prime}$ and would give $I_{2}(\eta)$ a value less than $\lambda_{i}$, which is a contradiction.

Writing $\mu$ and $\phi$ as symbols for the vectors $\left(\mu_{1}, \cdots, \mu_{\rho}\right)$ and $\left(\phi_{1}, \cdots, \phi_{\rho}\right)$, one sets up the form

$$
2 \omega_{i}=2 \omega_{0}-\lambda_{i} \eta \eta+2 \mu \phi,
$$

where the elements of $\mu$ are Lagrange multipliers to be determined. The determinant

$$
R_{i} \equiv\left|\begin{array}{ll}
\omega_{i z^{\prime} z^{\prime}} & \phi_{z^{\prime}} \\
\phi_{z^{\prime}} & 0
\end{array}\right|
$$

is readily found to equal $-|R|$. Hence $R_{i}$ is different from zero on $x_{1} \leqq x \leqq x_{2}$. 
Therefore it follows* that an arc which minimizes the integral (11) in the class $H_{i}$ must be a solution of the Euler-Lagrange system of equations

$$
\frac{d}{d x} \omega_{i z^{\prime}}-\omega_{i z}=0, \quad \phi=0 .
$$

These equations are the system

$$
J(\eta)+\lambda_{i} \eta+\mu_{\beta} \eta^{\beta}=0, \quad \phi=0 \quad\left(\mu_{\beta} \eta^{\beta} \equiv \sum_{\beta=1}^{p} \mu_{\beta} \eta^{\beta}\right),
$$

with the equations $d \mu_{\beta} / d x=0$ which say that the $\mu_{\beta}$ are constants.

For a quadratic form $2 \omega_{i}$ in the integrand it is readily seen that the second variation is a quadratic form with the same coefficients. The equations of variation $\dagger$ of the equations $\phi=0$ are the same equations since the $\phi$ are linear. The equations (12) are linear. It follows readily therefore that the accessory equations $\ddagger$ for the minimum problem under consideration are the Euler-Lagrange equations (12) themselves.

In the proof of the existence theorem of this section use will be made of the two following lemmas:

LEMMA $2 . \S$ The sets $\eta^{k}$ are linearly independent.

For, suppose $c_{\beta} \eta^{\beta}=0(\beta=1, \cdots, \rho)$. Then, for every $\eta^{k}$,

$$
0=\int_{x_{1}}^{x_{2}} c_{\beta} \eta^{\beta} \eta^{k} d x=\int_{x_{1}}^{x_{2}} c_{\gamma} \eta^{\gamma} \eta^{k} d x
$$

where $\gamma$ ranges over the indices for which $\eta^{k}, \eta^{\gamma}$ are in the same set $\eta^{i j}(j=$ $\left.1, \cdots, r_{i}\right)$. Therefore

$$
\int_{x_{1}}^{x_{2}} c_{\gamma} \eta^{\gamma} c_{8} \eta^{\delta} d x=0
$$

the range of $\delta$ being the same as that of $\gamma$. That is, $c_{\gamma} \eta^{\gamma}$ is identically zero and hence $c_{\gamma}=0$. A similar argument shows that all of the other coefficients $c_{\beta}$ are zero.

* Bliss, loc. cit. A, p. 11; Bolza, loc. cit., p. 558.

$\dagger$ Bliss, loc. cit. A, p. 4.

$\ddagger$ Bliss, loc. cit. A, p. 57; von Escherich, loc. cit., vol. 107, p. 1236.

$\S$ For the proof of Lemmas 2 and 3 it is convenient to use the notation of tensor analysis, along with the matrix notation. For example,

$$
c_{\beta} \eta^{\beta}=\sum_{\beta=1}^{\rho} c_{\beta} \eta^{\beta}
$$

stands for the vector $\left(c_{1} \eta_{1}{ }^{1}+\cdots+c_{\rho} \eta_{1}{ }^{\rho}, \cdots, c_{1} \eta_{n}{ }^{1}+\cdots+c_{\rho} \eta_{n}{ }^{\rho}\right)$. 
LEMMA 3. The Lagrange multipliers $\mu_{\beta}$ are zero for every solution $\eta(x)$ of (12) which satisfies the conditions

$$
\eta\left(x_{1}\right)=\eta\left(x_{2}\right)=0, \quad \zeta_{k}\left(x_{2}\right)=\int_{x_{1}}^{x_{2}} \eta^{k} \eta d x=0 .
$$

From the definition of $\zeta_{k}$ it follows also that $\zeta_{k}\left(x_{1}\right)=0$. For the proof of the lemma we have

$$
J(\eta)+\lambda_{i} \eta+\mu_{\beta} \eta^{\beta}=0, J\left(\eta^{k}\right)+\lambda^{\prime} \eta^{k}=0, \eta^{k}\left(x_{1}\right)=\eta^{k}\left(x_{2}\right)=0,
$$

where $\lambda^{\prime}$ is one of the constants $\lambda_{1}, \lambda_{2}, \cdots, \lambda_{i-1}$. Hence

$$
\eta^{k} J(\eta)-\eta J\left(\eta^{k}\right)+\left(\lambda_{i}-\lambda^{\prime}\right) \eta^{k} \eta+\mu_{\beta} \eta^{\beta} \eta^{k}=0,
$$

or, after some computation,

$$
\frac{d}{d x}\left(\eta^{k} \omega_{0 \eta^{\prime}}-\eta \omega_{0 \eta^{k^{\prime}}}\right)+\left(\lambda_{i}-\lambda^{\prime}\right) \eta^{k} \eta+\mu_{\beta} \eta^{\beta} \eta^{k}=0 .
$$

Integrating from $x_{1}$ to $x_{2}$ and using the hypotheses of the lemma, one obtains

$$
\mu_{\beta} \int_{x_{1}}^{x_{2}} \eta^{\beta} \eta^{k} d x=0 \quad(\beta, k=1, \cdots, \rho) .
$$

If the $\mu_{\beta}$ are not all zero, we find

$$
\int_{x_{1}}^{x_{1}} \mu_{\beta} \eta^{\beta} \mu_{\gamma} \eta^{\gamma} d x=0 \quad(\beta, \gamma=1, \cdots, \rho) .
$$

Therefore $\mu_{\beta} \eta^{\beta}$ is identically zero and hence by the preceding lemma $\mu_{\beta}=0$.

Theorem 4. There exists a set of $r_{i}$ linearly independent arcs $\eta^{i k}(x)(k=$ $\left.1, \cdots, r_{i}\right)$ in the class $H_{i}$ such that

$$
I_{2}\left(\eta^{i k}\right)-\lambda_{i} \int_{x_{1}}^{x_{2}} \eta^{i k} \eta^{i k} d x=0 \quad\left(k=1, \cdots, r_{i}\right) .
$$

Moreover $\lambda_{i}$ is definitely greater than $\lambda_{i-1}$.

For the proof one considers the problem of minimizing the integral (11) in the class $H_{i}$. As noted above, the Euler-Lagrange and the accessory system of differential equations are identical, namely

$$
J(\eta)+\lambda \eta+\mu_{\beta} \eta^{\beta}=0, \quad \phi=0,
$$

for $\lambda=\lambda_{i}$. This system is linear and homogeneous in the variables $z, z^{\prime}$, $z^{\prime \prime}, \mu_{\beta}, \mu_{\beta}^{\prime}$ and the determinant of the variables $z^{\prime \prime}, \mu_{\beta}^{\prime}$ is equal to the determinant of the matrix $R$. In $\S 1$, the solutions of such a system were considered 
and it was noted that a matrix $V$ can be uniquely determined whose columns are $n+\rho$ linearly independent solutions $z=(\eta, \zeta)$ of (13), whose elements all vanish at $x=x_{1}$, and such that the matrix of the $n+\rho$ corresponding fun ctions $\omega_{i z^{\prime}}$ is the identity matrix $I$ at $x=x_{1}$. Also if $\lambda$ is allowed to vary, the solutions of the system (13) are continuous functions of $\lambda$. The determinanteof the matrix $V$ is a Mayer determinant* for our minimizing problem, since its columns are $n+\rho$ linearly independent solutions of the accessory syst $\mathrm{m}$ which vanish at $x=x_{1}$. The vanishing of $|V|=\Delta\left(x, x_{1}, \lambda_{i}\right)$ for a value of $x$ between $x_{1}$ and $x_{2}$ implies $\dagger$ that there exists an admissible set $\xi$ of $n+\rho$ functions which substituted for $\eta, \zeta$ make the second variation of our minimum problem less than zero. Since this second variation is the same as the integral (11), we have a contradiction of the previous statement that $\lambda_{i}$ is the minimum value of $I_{2}(\eta)$ in the class $H_{i}^{\prime}$. Hence $\Delta\left(x, x_{1}, \lambda_{i}\right)$ cannot vanish for a value of $x$ between $x_{1}$ and $x_{2}$.

The proof that $\Delta\left(x, x_{1}, \lambda_{i}\right)$ must vanish at $x=x_{2}$ follows by an argument corresponding to that of Theorem 3 , if $\lambda_{0}$ is replaced by $\lambda_{i}, \Delta\left(x, x_{1}, \lambda_{0}\right)$ by $\Delta\left(x, x_{1}, \lambda_{i}\right)$ and the matrix $U$ by its corresponding matrix. Since $\Delta\left(x_{2}, x_{1}\right.$, $\left.\lambda_{i}\right)=0$, it follows that the columns of the matrix $V$ are linearly dependent at $x=x_{2}$.

If the rank of the matrix $V$ is $n+\rho-r_{i}$ at $x=x_{2}$, the equations

$$
V\left(x_{2}\right) c=0 \text {, }
$$

where $c=\left(c_{1}, \cdots, c_{n+\rho}\right)$ is independent of $x$, have $r_{i}$ linearly independent solutions $c \neq 0$, which define a fundamental system of solutions of these linear equations in terms of which all other solutions different from zero are linearly expressible. Moreover, for each such $c$ the vector $w=V c$ is a solution of the system of equations (12) with elements all vanishing at $x_{1}$ and $x_{2}$. The conditions of Lemma 3 are satisfied. Hence by Lemma 1 there exist $r_{i}$ linearly independent arcs

$$
\eta^{i k}, \zeta_{1}^{i k}, \cdots, \zeta_{p}^{i k} \quad\left(k=1, \cdots, r_{i}\right)
$$

of the class $H_{i}$ for which the $\eta^{i k}$ satisfy equation (10) with $\lambda_{0}$ replaced by $\lambda_{i}$.

To prove $\lambda_{i}>\lambda_{i-1}$, it is first shown that no $\eta^{i k}$ can be a linear combination of the $\eta^{i-1, k}$. This follows from the proof of Lemma 2 if we let the sets $\eta^{k}$ include the $\eta^{i j}$ as well as $\eta^{0 i}, \cdots, \eta^{i-1, j}$. In the class $H_{i-1}$ the only sets $\eta$ making

* Bliss, loc. cit. A, p. 63; Bolza, loc. cit., p. 611.

$\dagger$ Bliss, loc. cit. A, p. 59; Bolza, loc. cit., p. 617. 


$$
I_{2}(\eta)-\lambda_{i-1} \int_{x_{1}}^{x_{2}} \eta \eta d x=0
$$

are the sets $\eta^{i-1, k}\left(k=1, \cdots, r_{i-1}\right)$ and linear combinations of them, as was found in the preceding pages. As just shown above, no $\eta^{i k}$ can be such a linear combination. But $\eta^{i k}$ satisfies the relation

$$
I_{2}(\eta)-\lambda_{i} \int_{x_{1}}^{x_{2}} \eta \eta d x=0 .
$$

Hence $\lambda_{i} \neq \lambda_{i-1}$, and since $\lambda_{i}$ cannot be less than $\lambda_{i-1}$ one has $\lambda_{i}>\lambda_{i-1}$. This completes the proof of Theorem 4 , and one readily sees that the following corollary is also true:

COROLIARY. There exists a set of $r_{i}$ linearly independent arcs in the class $H_{i}^{\prime}$ for which the integral $I_{2}(\eta)$ takes the value $\lambda_{i}$.

BROWN UNIVERSITY,

Providence, R. I. 